\begin{tabular}{|l|l|l|l|}
\hline Eiszeitalter u. Gegenwart & $\mathbf{2 9}$ & $\begin{array}{l}9-22 \\
3 \mathrm{Abb} .\end{array}$ & Hannover 1979 \\
\hline
\end{tabular}

\title{
Verbreitung, Ursachen und Füllung glazial übertiefter Talabschnitte an Beispielen in den Ostalpen.
}

\author{
Dirk VAN Husen *)
}

Glacial erosion, depression, glacial valley, moraine, aggradation, fluvioglacial, extent, longitudinal profile, geological section

North-Austrian Alps (Salzach, Traun Valley), Central-Austrian Alps (Enns Valley), Salzburg

Kurzfassung: Im untersuchten Raum konnte ein Zusammenhang zwischen dem Auftreten ausgedehnter glazial übertiefter Becken und der Ausdehnung aktiver Gletscherzungen gefunden werden. Jene liegen im Zehrgebiet dieser und sind möglicherweise auf die hohe Fließgeschwindigkeit des Eises und dessen starke Anreicherung mit Moränenschutt zurückzuführen.

Die Auffüllung der Becken erfolgte sehr rasch im beginnenden Spätglazial und war zur Zeit der Wiederbewaldung bereits abgeschlossen. Dabei kamen petrographische Unterschiede im Einzugsgebiet der Flüsse stark zum tragen.

\section{[Distribution, Reasons and Refill of Glacially Deepened Valley-Sections by Examples of the Eastern Alps.]}

A bstract: In the investigated area a relationship between the appearance of widespread glacially deepened basins and the extent of active glacier terminus could be found. Those lie in the ablation area of these and probably are due to the high velocity of flowing ice and its increasing heavy charge of morainic debris.

At the beginning of Late Glacial Time the refill of the basins was very fast. It was already achieved at the time when the woods returned. Petrographic differences in the drainage area of the rivers were of important influence.

\section{Einleitung}

Die vorliegende Arbeit stellt die etwas erweiterte Fassung des Vortrages gleichen Titels im Rahmen des Edith-Ebers-Symposiums in Rosenheim dar.

An drei durch künstliche Aufschlüsse und Detailkartierungen des Autors einigermaßen bekannten Beispielen aus den Ostalpen wird die Gestaltung und Verbreitung der glazial übertieften Becken und deren Füllung dargelegt.

Zweck dieser Arbeit ist primär die Erfassung alter, bis heute nur in einzelnen verstreuten Arbeiten niedergelegter und in neuerer Zeit hinzugekommener Daten. Darüber hinaus soll auch der Versuch unternommen werden, aus der regionalen Verteilung in Bezug auf die Erstreckung der Lokalgletscher und Eisstromnetze zu einer möglichen Erklärung der Entstehung der größeren glazial übertieften Wannen zu gelangen.

An dieser Stelle möchte ich allen im Text namentlich genannten Personen, die mir noch unpublizierte neue Daten zur Verfügung stellten, herzlich für ihr Entgegenkommen danken.

\section{Salzburger Becken}

Das Zungenbecken des Salzachgletschers zwischen Golling im S und den Endmoränen im N stellt einen Modellfall von Stamm- und Zweigbecken dar (Penck \&

*) Anschrift des Verfassers: Univ.-Doz. Dr. Dirk v a n $\mathrm{H}$ u se n, Geologisches Institut d. Technischen Universität, Karlsplatz 13, A-1040 Wien. 
BrÜCKNer 1909: $157 \mathrm{ff}$.). Das Stammbecken selbst ist eine ca. 2-3 km breite Rinne, die am morphologischen Nordrand der Alpen endet (Abb. 1).

Im nördlichen Abschnitt war die Tiefe des Beckens durch einige Tiefbohrungen schon länger bekannt (PREY 1959). Der Untergrund wurde in $262 \mathrm{~m}$ Tiefe ca. $1 \mathrm{~km}$ weiter nördlich bereits in $198 \mathrm{~m}$ erbohrt.

O'stlich dieser beiden Bohrungen steigt der Untergrund, wie aus anderen Bohrungen bekannt ist, steil zum Mönchsberg hin an. Durch die zwei Bohrungen der Stieglbrauerei, die nahe der Beckenmitte stehen, scheint die absolute Tiefe des Beckens auf der Höhe von Salzburg gut erfaßt zu sein, obwohl Tiefenaufschlüsse weiter westlich fehlen.

Weiter im S gab die 1976 abgeteufte Bohrung Vigaun V1 der OMV A.G. Wien einen weiteren Fixpunkt der Tiefenlage der Felssohle. Die für die Fragestellung der Übertiefung und Füllung des Beckens aussagefähigen Daten dieser Bohrung wurden mir freundlicherweise von Direktor Dr. Kröll zur Verfügung gestellt. Eine genauere Auswertung der Ergebnisse wird noch durch die OMV A.G. Wien erfolgen.

Die am südlichen Rand der Ortschaft Vigaun stehende Bohrung erreichte nach $338 \mathrm{~m}$ den Untergrund (Oberalmer Schichten) und dürfte unter der Annahme eines U-förmigen Talquerschnittes ebenso wie die bei Salzburg bereits weitgehend die volle Tiefe des Bekkens im Raum Hallein angeben.

Noch weiter im S wurde bei Golling an der Mündung der Lammer in die Salzach im Zuge von Grundwassererschließungsarbeiten der Fels in $161 \mathrm{~m}$ Tiefe erbohrt (freundl. Mitteilung Dr. H. BRANDECKER). Da aber unmittelbar nordwestlich davon der Felsuntergrund im Bereich des Flusses noch zutage tritt (BrandeCKer 1974: Taf. I) handelt es sich hier wahrscheinlich eher um eine Fortsetzung der sicher subglazial angelegten Tiefenrinne der Salzach in Fortsetzung der Salzachöfen als bereits um den glazial übertieften Beckenuntergrund.

Aus den vorliegenden Bohrungen und Vergleichen mit anderen Becken läßt sich der Verlauf des glazial übertieften Felsuntergrundes des Stammbeckens des Salzachgletschers im großen rekonstruieren (Abb. 1).

Die glaziale Übertiefung setzt südlich Golling am Nordrand des Ofenauer Berges ein. Der Ansatzpunkt ist hier durch zwei Umstände gegeben. Einerseits überwand das aus dem Pongau nach $\mathrm{N}$ abfließende Eis den aus dickbankigem Dachsteinkalk aufgebauten Riegel zwischen Hagen und Tennen Gebirge, der auch eine deutliche Querschnittsverengung darstellt, mit einem starken Gefällsbruch. Dieser ist zumindest für die Würmeiszeit durch die riesigen Strudeltöpfe am Ofenauer Berg (HASERODT 1965) belegt.

Andererseits stellt die steil nach $\mathrm{N}$ abtauchende Schichtung des Dachsteinkalkes mit ihrer leichter ausräumbaren Juraauflage eine günstige Konstellation für eine glaziale Übersteilung der Stufe dar.

In der weiteren Folge sinkt dann der Felsuntergrund bald auf jene Tiefe ab, die durch die Bohrung Vigaun erfaßt ist und wahrscheinlich auch die generelle Tiefenlage der Bekkensohle im mittleren Beckenabschnitt markiert.

Über den genaueren weiteren Verlauf der Felssohle gibt es aber leider keine Aufschlüsse, da Bohrungen fehlen. Das übertiefte und heute durch junge Sedimente verfüllte Becken verläuft als $2-3 \mathrm{~km}$ breiter, grabenartiger Streifen weiter nach NNW. Diese Form war der Grund für Überlegungen einer praeglazialen tektonischen Anlage des Beckens (zusammenfassende Darstellung bei DeL NEGRo 1966: 171), die vom Gletscher nur noch überformt worden ist. 

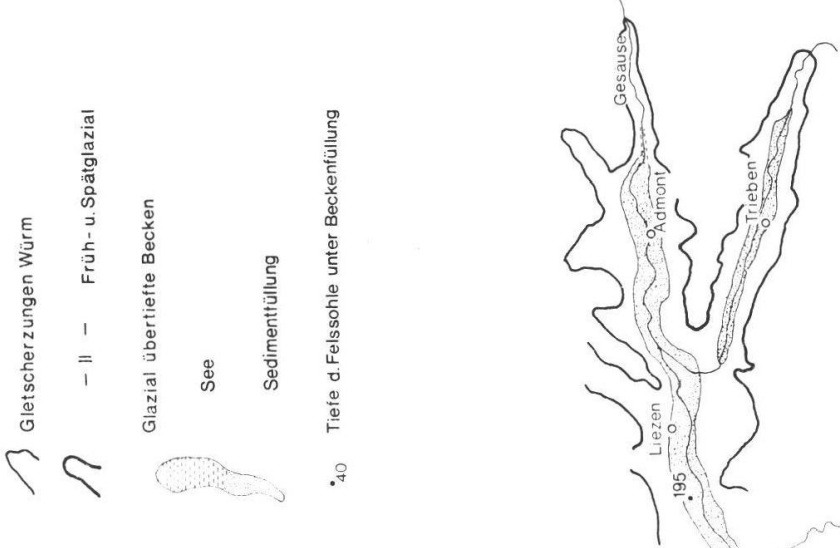

泀

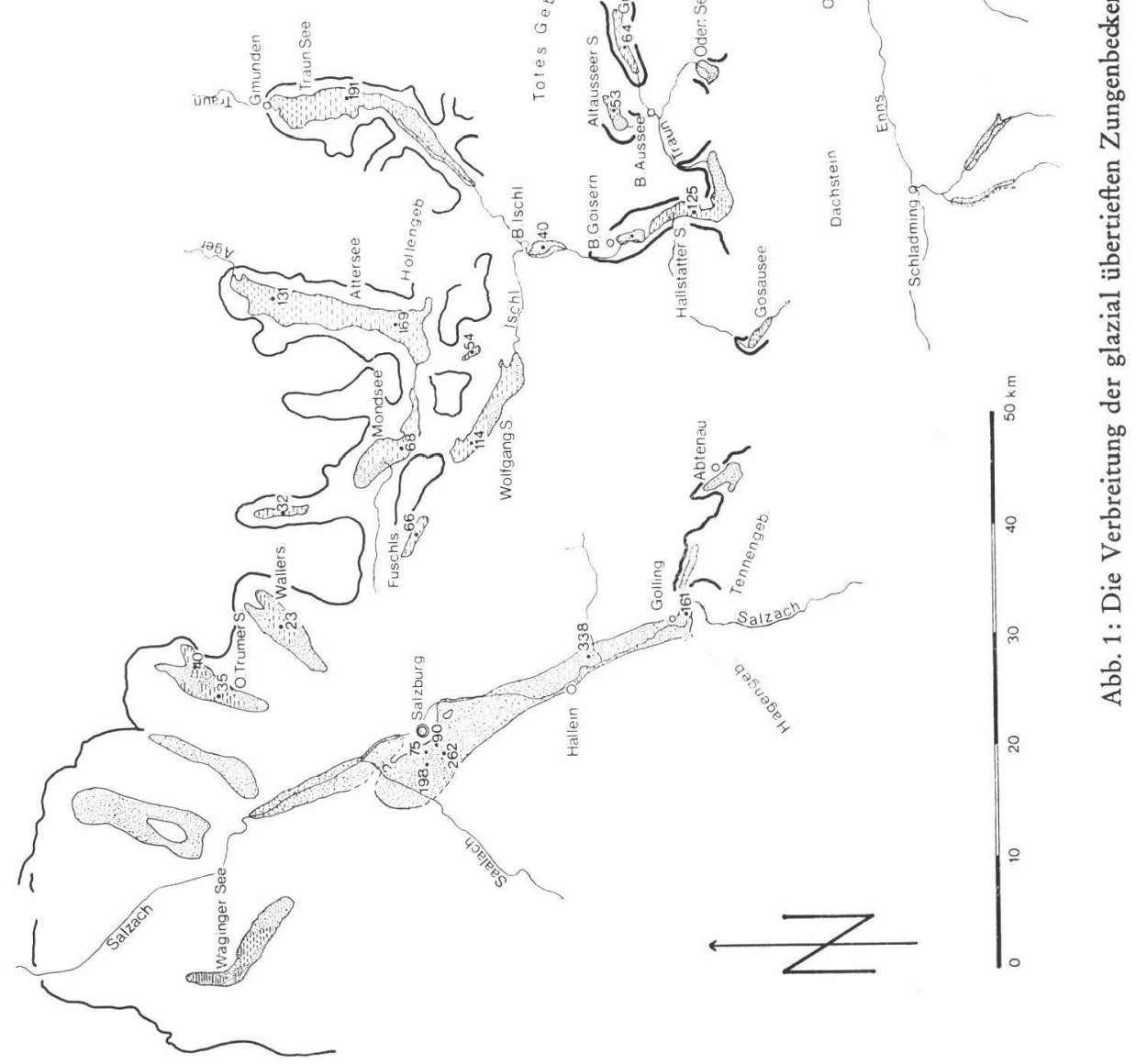


Abgesehen von tektonisch bedingten Voraussetzungen, die bereits zur Anlage des praeglazialen Salzachtales führten, ist aber die heutige Form und Sohlenlage des Beckens doch durchaus auf die Gletscherarbeit zurückzuführen. Es ist wahrscheinlich durchwegs in die weitgehend eben gelagerten, gleichmäßig ausgebildeten Oberalmer Schichten eingesenkt, die auch in der Bohrung Vigaun V1 im Liegenden der quartären Sedimente angefahren wurden.

Daraus, und im Vergleich mit der Sohlenentwicklung anderer Becken (Trauntal), wird wahrscheinlich zumindest in diesem Bereich, abgesehen von kleineren Schwankungen, mit einem weitgehend ebenen Verlauf der Beckensohle und steilen Flanken zu rechnen sein.

Die Tiefenrinne verläuft dann westlich der inselartig aus den jüngeren Aufschüttungen aufragenden Hügel um Hellbrunn und Rainberg-Mönchsberg weiter, Richtung Max GlanSietzenheim.

Hier wurde der Untergrund in den zwei Bohrungen der Stieglbrauerei angefahren, über die PREY (1959) ausführlich berichtete.

Die südliche beim Kugelhof erreichte in $262 \mathrm{~m}$ Tiefe den Felsuntergrund und zeigt damit an, daß sich die generelle Höhenlage der Felssohle $16 \mathrm{~km}$ nördlich Vigaun innerhalb der Kalkalpen noch nicht wesentlich verändert hat. In der ca. $1 \mathrm{~km}$ weiter nördlich liegenden Bohrung wurde aber bereits in $198 \mathrm{~m}$ Mergel, wahrscheinlich Flysch (PREY 1959: 221) im Untergrund erbohrt. $\mathrm{Ob}$ es sich dabei um eine örtliche Aufragung, oder, was wahrscheinlicher scheint, um ein Ausheben der Rinne gegenüber ihrem Nordende handelt, kann nicht mit Sicherheit gesagt werden.

In der geradlinigen Fortsetzung der Rinne treten dann NW Salzburg die Würmgrundmoräne und in der weiteren Folge auch ältere quartäre Sedimente auf. PREY (1959) parallelisiert auf Grund des Geschiebeinhalts erstere mit der Grundmoräne, die in den Bohrungen unmittelbar dem Felsuntergrund aufliegt. Daher scheint es wahrscheinlich, daß durch die beiden Bohrungen das Ende des Stammbeckens zur Würmeiszeit mit dem nach $\mathrm{N}$ aushebenden Untergrund erfaßt wurde. Es ist aber genau der Bereich, wo der Salzachgletscher aus dem relativ engen inneralpinen Tal ins Vorland austritt und sich im W durch den Saalachgletscher behindert hauptsächlich nach E ausbreitete. Dadurch kam es sicher zu einer deutlichen Abnahme der Fließgeschwindigkeit und einem Erlahmen der Tiefenerosion gegenüber dem kanalartigen Stammbecken südlich von Salzburg.

Wie mächtig hier die Würmgrundmoräne ist, und ob die Rinne in früheren Eiszeiten noch etwas weiter nach $\mathrm{N}$ reichte, kann nicht gesagt werden, da Aufschlüsse völlig fehlen.

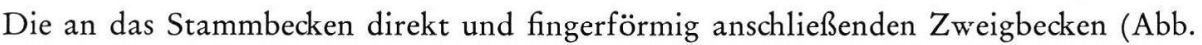
1) stellen teils subglaziäre Rinnen (z. B. Oichental, WEINBERgER 1955: 11) oder seichtere Wannen in den quartären Sedimenten dar, über deren Übertiefung kaum etwas bekannt ist. Einige Hinweise geben die Wassertiefe der Seen (Waller-, Matt-, Obertrumer See).

\section{Auffüllung}

Mit Ende des Hochglazials wurde der Bereich der Zweig- und des Stammbeckens sehr rasch eisfrei. Die Becken füllten sich mit Seen, die teilweise mit Schottern und Schluff verfüllt wurden (Ibmer Moor) oder noch bis heute erhalten sind. Die Entwicklung dieser Seen in ihrer Beziehung zum See des Stammbeckens wurde von Weinberger (1955: 23) und Del-Negro (1966: $203 \mathrm{ff}$.) genau beschrieben.

Die Sedimente des Stammbeckens sind durch die erwähnten Tiefenbohrungen der Stieglbrauerei (PREY 1959), der OMV A.G. bei Vigaun und umfangreiche Grundwasseruntersuchungen (BRANDECKER 1974) soweit bekannt, daß der Versuch ein Gesamtbild der Verfüllung zu entwerfen, berechtigt scheint (Abb. 2). 


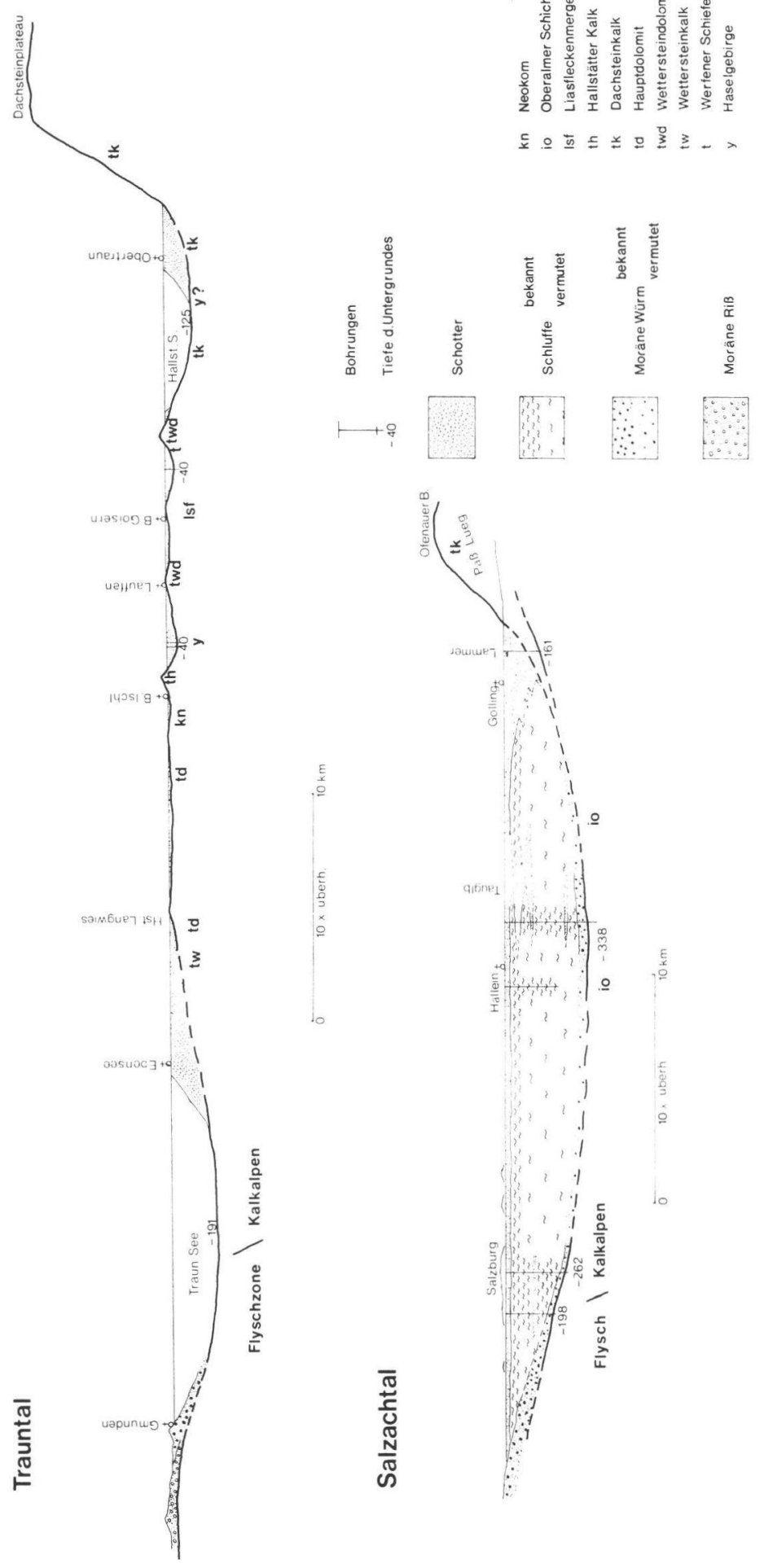


Das hangendste Sediment im Becken bilden im Mittel $20 \mathrm{~m}$ mächtige Schotter, die von $\mathrm{S}$ nach $\mathrm{N}$ mit stetig abnehmender Mächtigkeit den ganzen Raum überziehen. Sie gehen in einer mehrere Meter mächtigen Übergangsschicht in die liegenden Schluffe über, mit denen sie sich auch randlich verzahnen. Sie stellen die zu einer letztlich geschlossenen Schotterdecke verwachsenen, groben Einschwemmungen der Salzach und ihrer Nebenbäche nach Abschluß der Seephase im Stammbecken dar.

Darunter liegen in den Bohrungen westlich des Mönchsberges mächtige, schluffige Seesedimente, die fast die ganze Mächtigkeit der Beckenfüllung ausmachen. Sie wurden in den drei Tiefbohrungen im Bereich um $140 \mathrm{~m}$ von Feinsandlagen mit eingestreuten kleinen Geröllen unterbrochen, die Prey (1959: 220) im Gegensatz zu Stummer (1947) aus mehreren Gründen auf Einschwemmungen vom Rand oder auch von schwindendem Gletschereis deutet und nicht als Ablagerungen älterer Eiszeiten, da ja auch Hinweise auf höhere Lagerungsdichte der liegenden Schluffe fehlen.

Eine Interpretation der in den Schluffen auftretenden Picea-Pollen als Hinweis auf Sedimentreste älterer Eiszeiten kann nicht vorgenommen werden. Wie an den Vorkommen älterer Seesedimente ersichtlich ist, bestand nach dem Abschmelzen des Mindel- und Rißgletschers jeweils ein See mit etwas höherem Wasserstand als nach dem Würmhochglazial. $\mathrm{Da}$ aber auch in diesen Seen ähnliche Sedimentationsraten und daher ähnlich rasche Verfüllung wie nach der Würmeiszeit anzunehmen ist, ist ein autochthones Vorkommen von Picea-Pollen auch in diesen Sedimenten nicht zu erwarten.

Im Liegenden der Schluffe folgt dann über der Felssohle dichte Grundmoräne in Verbindung mit Sand- und Schlufflagen, die PrEY (1959: 220) durch den Vergleich der Geschiebezusammensetzung für die des Würmgletschers ansieht.

Prinzipiell den gleichen Aufbau der Beckenfüllung zeigen die Bohrungen in Kaltenhausen und bei Vigaun im Raum Hallein.

In der Bohrung Vigaun wurde im Liegenden ein rund $100 \mathrm{~m}$ mächtiges Paket von dichter Grundmoräne und Schottern, im Hangenden der Oberalmer Kalke angefahren. Darüber folgen wieder die glimmerreichen, schluffigen Seesedimente, die aber von drei, 20, 7 und $40 \mathrm{~m}$ mächtigen, schlufführenden Schotterlagen unterbrochen werden. Bei diesen handelt es sich wahrscheinlich um Einschüttungen (subaquatische Gleitungen vom Schwemmkegel der Taugel), da sie sich durch höhere Wasserführung und deutlich niedrigere seismische Geschwindigkeit von der liegenden Grundmoräne (ca. 3000/sec.) unterscheiden.

Neben diesen weiter in das Becken ausgreifenden Einschüttungen sind durch Erschließung von Grundwasservorkommen mächtigere Deltaablagerungen, die sich mit den feinkörnigen Sedimenten verzahnen, auch an den Mündungen der kleineren Seitenbäche erschlossen worden (BRANDECKER 1974: $12 \mathrm{ff}$.).

Ebenso konnte im Raum Golling eine ausgedehntere, mächtige Deltabildung der Salzach, Lammer und des Torrener Baches erschlossen werden (mündl. Mitt. Brandecker).

Zeugen älterer, weiter nach $\mathrm{N}$ ausgreifender Deltabildungen stellen die konglomerierten Deltaschotter westlich Golling (Torrener Nagelfluh) dar. Sie und ähnliche Bildungen, die im ganzen Beckenbereich randlich auftreten, sind Zeugen von Stauseen, die unmittelbar nach dem Eisrückgang früherer Eiszeiten mit höherem Wasserspiegel als der im Spätwürm das Becken von Salzburg erfüllten.

Die Auffüllung des letzteren erfolgte wahrscheinlich sehr rasch, wie aus der geringen räumlichen Ausdehnung der sich mit der schluffreichen Beckenfüllung verzahnenden, grobkörnigen Deltabildung der einmündenden Bäche ablesen läßt. 
Als einen weiteren Hinweis für eine solche rasche Sedimentation im Vergleich mit der Füllung im Ennstal kann noch die sehr geringe Pollenkonzentration in den Sedimenten (PREY 1959: $221 \mathrm{f}$.) und das Fehlen anderer Fossilien angesehen werden. Auf alle Fälle war die Füllung zur Zeit der Bildung der höchsten Terrasse (Friedhofsterrasse) beendet.

$\mathrm{Ob}$ die Füllung des Beckens, abgesehen von den randlich auftretenden sicher älteren Sedimenten, zur Gänze aus denen des Würm-Spätglazials besteht oder auch solche älterer Eiszeiten im Untergrund noch erhalten sind, kann nicht sicher beantwortet werden. Obwohl das völlige Fehlen von sicheren Grundmoränenablagerungen innerhalb der Schluffe in allen Bohrungen kein absolutes Kriterium (Diskussionsbemerkung Prof. Dr. B. FreNZEL) darstellt, so fehlen auch Hinweise auf höhere Lagerungsdichte liegender Sedimentanteile, die auf eine Vorbelastung durch Gletscherzungen hindeuten würden. Darüber hinaus wäre ihre Erhaltung in dem engen, trogartigen Becken mit seinen geradlinigen Flanken und Verlauf, das vom Gletscher mit großer Geschwindigkeit durchflossen wurde, schwer vorstellbar.

\section{Trauntal}

Im Gegensatz zum Salzachgletscher erreichte der Traungletscher in der Würmeiszeit nur noch den Alpennordrand und trat in den vorangegangenen Eiszeiten nur wenig ins Vorland ein. Der Grund für die wesentlich geringere Ausdehnung liegt hauptsächlich darin begründet, daß der Traungletscher in seinem Einzugsgebiet nur auf die niedrigeren Nördlichen Kalkalpen beschränkt blieb und kaum Zugang aus den Zentralalpen hatte. Überdies spaltete sich der Eisstrom im Raum Bad Ischl-St. Gilgen in vier Gletscherzungen auf.

Dadurch kam es zur Ausbildung von vier Zungenbecken, die die großen Salzkammergutseen beinhalten, an denen Erstreckung und Ausformung jener recht gut ablesbar ist (Abb. 1 und 2).

Im Bereich des unteren Trauntales, das zwischen Mitterweissenbach und Ebensee einer großen Störung nach N wahrscheinlich der von GEYER (1917) vermuteten Blattverschiebung folgt, setzt die Übertiefung nordwestlich der Felsschwelle beim Bhf. Langwies (vaN Husen 1977) ein. Die Eintiefung erfolgte im Bereich von massigem Hauptdolomit, Wettersteinkalk und Dachsteinkalk, innerhalb derer sie noch ihren höchsten Wert von $191 \mathrm{~m}$ erreicht (Abb. 2). Erst nach der Grenze Kalkalpen-Flysch hebt das Becken zu den Würmendmoränen von Gmunden hin aus. Auffällig ist das trogartige Querprofil mit steilen Wänden und einer breiten, sedimentbedeckten Sohle, die über den ganzen See zu verfolgen ist und erst im Bereich des Flysches in eine dann bald aushebenden Rinne übergeht.

Im Bereich der Zunge des Attersees, deren Ernährung hauptsächlich durch das Mitterweißenbachtal und die Schwarzensee Furche erfolgte (VAN HuSEN 1977), setzt die Übertiefung an der Grenze Kalkalpen-Flysch ein, dürfte aber auch im Äußeren Weißenbachtal bereits wirksam sein. Die auffällige Bindung des Einsatzes der glazialen Tiefenerosion am Südende des Attersees hat seinen Grund darin, daß der Traungletscher hier in ein erst E-W an der Grenze Flysch-Kalkalpen, dann nach $N$ verlaufendes Tal (ähnlich der Aurach am Nordrand des Höllengebirges) übertrat und die vorhandene Gefällsstufe überarbeitete.

Die Form des Beckens des Attersees ist auch die einer steilwandigen Wanne mit einer weitgehend ebenen Sohle, die erst am nördlichen Seeende zu den Endmoränen hin rasch aushebt.

Ein Ast des Traungletschers verlief das Ischltal aufwärts über St. Gilgen bis zum Fuschlsee. Der glazial übertiefte Bereich beginnt im Ischltal auf der Höhe von Strobl. Die 
Erosion setzt somit erst mitten in dem von Gosau und Flyschgesteinen gebildeten Talbereich ein, ohne daß ein Zusammenhang mit Strukturen oder petrographischen Unterschieden zu erkennen wäre.

Ist die Haupterosionswirkung im Ostteil des Wolfgang Sees eher rinnenförmig auf den Nordrand beschränkt, so ist nach der Verdeckung durch das Zinkenbachdelta im Westteil bereits wieder das steilwandige Querprofil mit einer sedimentbedeckten Sohle ausgebildet. Aus dem Bereich St. Gilgen floß ein nicht unerheblicher Teil des Eises gegen $\mathrm{N}$ ab. Der verbleibende Rest erfüllte noch das Becken des Fuschlsees. Das nach $\mathrm{N}$ abfließende Eis vereinigte sich mit dem am Südende des Attersees abgespaltenen, nach W fließenden Eisstrom. Vereinigt schürften sie die flache Wanne des Mondsees aus.

In der weiteren Folge erfüllte diese Gletscherzunge noch das Becken von Talgau und nach seitlichem Überfließen einer R/W Interglaziale Sedimente (KLAus 1975) enthaltenden Schwelle nördlich Mondsee das des Irrsees.

Neben diesen im Zungenbereich liegenden, langgestreckten Wannen, die auffälligerweise um den Bereich der Schneegrenze während der beiden letzten Eiszeiten ihren Anfang haben, treten im Trauntal noch ähnliche Formen im $S$ auf.

So stellt der fjordartig am Nordrand des Dachsteinmassivs eingesenkte Hallstätter See eine langgestreckte Wanne mit steilen Wänden und ebener Sohle dar. Sie ist (max. Tiefe $125 \mathrm{~m}$ ) bis auf einen kleinen Bereich (wo möglicherweise Haselgebirge auftritt) südlich Hallstatt (Abb. 2) durchwegs in Dachsteinkalk eingetieft.

An der Südostabdachung des Toten Gebirges stellen der Altausseer See und der Grundlsee gleiche Formen dar. Ihre Tiefe scheint hauptsächlich von der Größe des Einzugsgebietes der Gletscherzungen abzuhängen.

Einerseits könnten diese Seebecken als glazial übertiefte Bereiche unter den bereits praeglazial vorhandenen hohen Steilstufen am Rand der Dachsteinkalkplattformen angesehen werden, was wahrscheinlich für die kleinen, sehr tiefen Becken (Toplitz und Ht. Gosausee), kaum aber bei den weiter entfernten Bereichen (Grundlsee) zutreffen dürfte.

Andererseits liegen diese Becken aber alle im Zungenbereich kräftiger Lokalgletscher, wie sie während der Würmvereisung zweimal über längere Zeit bestanden (vaN HusEN 1977). Demnach könnten diese Bereiche durchaus als Zungenbecken von Gletschern angesehen werden, die dieser wahrscheinlich auch während früherer Eiszeiten relativ rasch erreichbaren Gletschergröße entsprechen.

Zwischen den übertieften Bereichen im S des Einzugsgebietes der Traun und den Zungenbecken im N sind im Trauntal durch die Bohrungen der Salinenverwaltung noch kleinere Becken von rund $40 \mathrm{~m}$ Tiefe zwischen den obertägig verfolgbaren Schwellen bekannt (Abb. 2).

Die Verbreitung der Becken und Schwellen zeigt bis auf das Becken südlich Bad Ischl (über Haselgebirge) keinen Zusammenhang mit der petrographischen Zusammensetzung und der damit verbundenen Erodierbarkeit des Untergrundes, wobei auch weiche Gesteine (Liasfleckenmergel) als Schwellenbildner auftreten.

Ebenso wirkt sich auch der Zusammenfluß großer Eisströme wie des vom Dachstein (über den Hallstätter See) mit dem vom Toten Gebirge und dem Mitterndorfer Becken über den Pötschenpaß nicht in einer Steigerung der Erosionsleistung am Gletschergrund aus.

An der Einmündung des Eisstromes des Rettenbachtales bei Bad Ischl konnten sich die weichen Neokomsandsteine und weiter westlich Gosaukonglomerate und Mergel erhalten, die zu langgestreckten Rundhöckern geformt wurden. 


\section{Auffüllung}

Die Auffüllung der glazial übertieften Becken des Trauntales erfolgte hauptsächlich durch die Traun, wobei die Nebenbäche nur ganz selten eine wesentliche Rolle spielten.

Die kleineren Wannen bei Bad Ischl und Bad Goisern wurden, wie aus den Bohrungen der Salinenverwaltung bekannt ist, mit schluffreichen Schottern und Sanden, sicher unmittelbar nach dem Eisrückzug verfüllt.

In den beiden großen Wannen, die von der Traun durchströmt werden (Traun und Hallstätter See), kam es zur Ausbildung von Deltaablagerungen bei Ebensee und Obertraun. Die Bildungen dieser beiden ausgedehnten Aufschüttungen erfolgte wahrscheinlich sehr rasch und in der Hauptsache noch bevor sich eine geschlossene Gehölzvegetation mit Ende der Böllingschwankung (VAN Husen 1977) im Tal der Traun ausbreiten konnte und damit die Schuttanlieferung stark reduziert wurde. Eine Erfüllung der Seebecken mit Schluffen unterblieb, da die Traun im Gegensatz zur Salzach und Enns als rein kalkalpiner Fluß eine wesentlich geringere Belastung mit Feinstoffen aufweist.

Über den inneren Aufbau dieser Deltaschüttungen und ob darin auch ältere Sedimente eingeschlossen sind, kann keine Aussage getroffen werden, da Bohrungen fehlen. Da aber im Trauntal, wie eine Detailkartierung 1 : 10000 (VAN HuSEN 1977) zeigte, auch in gegen Gletschererosion geschützten Lagen nur ganz geringe Reste von Sedimenten älterer Eiszeiten erhalten sind, möchte ich eher auf das Fehlen solcher Sedimente gerade in besonders stark und rasch durchflossenen Bereichen schließen.

In den anderen Seebecken kam es an der Einmündung der Bäche zur Ausbildung durchwegs kleinerer Deltas. Eine Ausnahme bildet nur das Delta des Zinkenbaches am Südufer des Wolfgang-Sees.

\section{Ennstal}

Im Mitterennstal ist der Bereich der übertieften Felssohle nicht so deutlich zu erfassen wie in den beiden vorher beschriebenen Tälern (Abb. 3).

In der Längstalfurche ist als sicher übertiefter Abschnitt der Raum zwischen Gesäuse und Grimming anzunehmen, dessen Felssohle durch die Tiefbohrung bei Wörschach in $195 \mathrm{~m}$ auf $444 \mathrm{~m}$ üb. NN in ungefähr der gleichen Höhe wie bei Hieflau (443 m üb. NN) unterhalb des Gesäuses festgelegt ist (Abb. 3).

Ebenso dürfte der Abschnitt des Paltentales zwischen Strechau und Tregelwang eine glaziale Übertiefung aufweisen (Abb. 1).

Diese Talabschnitte decken sich aber ebenso wie im Trauntal mit dem Zehrgebiet des würmzeitlichen Gletschers, der nur noch als schmale Zunge ins Gesäuse eindrang und auf dem Sattel der Buchau seine Endmoränen hinterließ (van Husen 1968).

Neben diesen Zungenbecken finden sich im Einzugsgebiet der Enns in den großen, bis zum Hauptkamm der Niederen Tauern zurückgreifenden Seitentälern auffallende langgestreckte Talweitungen. Sie sind alle durch im Fels angelegte, längere, enge und teilweise schluchtartige Talstrecken vom Ennstal getrennt. In zwei dieser Talweitungen (Sölktäler) wurden für eine Kraftwerksgruppe der STEWEAG Sondierbohrungen durchgeführt, deren Ergebnisse mir Prof. Dr. E. Clar freundlicherweise zur Verfügung stellte.

Es zeigte sich, daß im Tal des Kleinsölkbaches $450 \mathrm{~m}$ südlich der projektierten Sperrenstelle, die ein über die ganze Talbreite geschlossenes Felsprofil aufweist, die Felssohle bereits 41,4 m übertieft ist. Ebenso wurde im Tal des Großsölkbaches südlich der im Fels angelegten Engstrecke ein Absinken der Felssohle festgestellt, ohne diese aber zu erreichen. 
Längsprofil

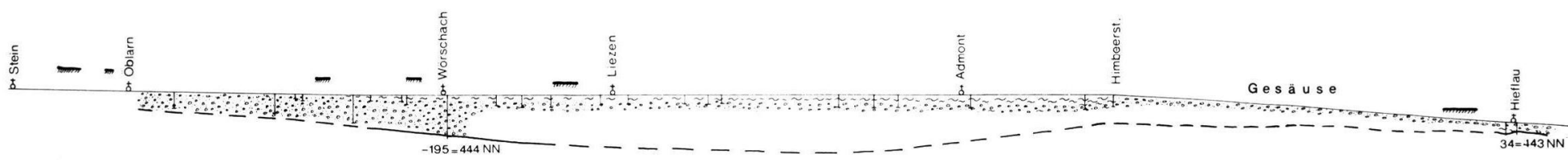

- $10 \times \overline{\text { uberh }} \longrightarrow 20 \mathrm{~km}$

Querprofil

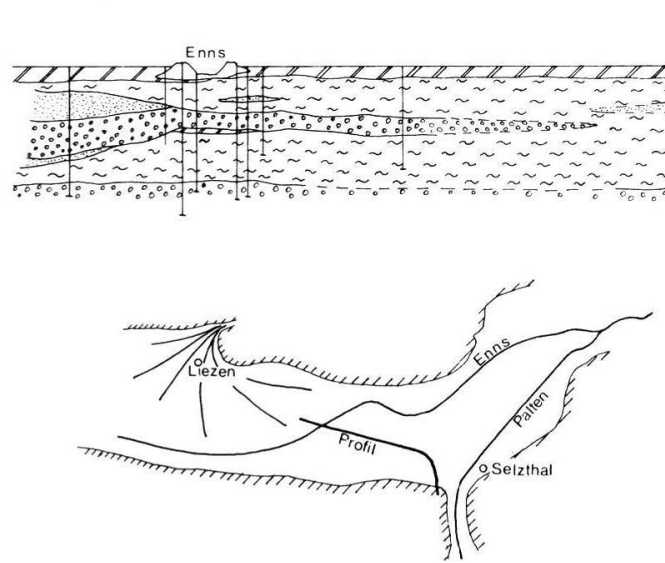

Bohrung
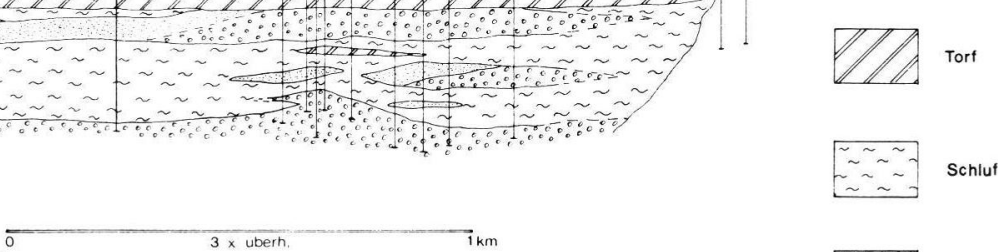

Schotter/Sand

Praegl Talboden

Abb. 3: Läng s- und Querprofil durch das Ennstal. 
Auf Grund dieser Beobachtungen scheint es berechtigt zu sein, auch für die gleich gestalteten Talabschnitte der übrigen Täler eine glaziale Übertiefung in den Talweitungen anzunehmen.

Ein Zusammenhang mit leichter ausräumbaren Gesteinen kann im Bereich der Nordabdachung der Niederen Tauern mit ihren streng E-W verlaufenden Gesteinszügen und Strukturen nicht gegeben sein.

Im Gegensatz zu den übertieften Bereichen im südlichen Trauntal fehlen hier auch die praeglazial angelegten Gefällsstufen, die für die Bildung dieser Becken herangezogen werden könnten. Es handelt sich demnach, wie ich annehmen möchte, auch hier um Zungenbecken kräftiger Lokalgletscher der einzelnen Täler, die auch im gleichen Größenverhältnis zum Eisstrom des Hochglazials stehen, wie die des Trauntales. Die Zeit der endgültigen Formung dürfte demnach ebenso die gleiche wie dort sein.

\section{Auffüllung}

Das Zungenbecken des Ennstales stellt sich heute nach seiner Füllung als sehr gefällsarme, hauptsächlich von mühsam meliorierten Sümpfen und Mooren bedeckte Talniederung dar, in der nur die Schwemmkegel der seitlichen Zubringer den Siedlungsraum bilden.

Die obersten $50 \mathrm{~m}$ der Sedimente des Zungenbeckens sind vor allem durch die Untersuchungen für einen Grundwasserspeicher und in jüngster Zeit durch die Sondierungen für den Bau der Pyhrn-Autobahn recht gut bekannt geworden.

Die Ergebnisse für den Autobahnknoten Selzthal der Pyhrnautobahn stellte mir freundlicherweise Dr. O. Homann zur Verfügung. Sie zeigen unter einer mehrere Meter mächtigen Torfauflage für die obersten $20-25 \mathrm{~m}$ der Beckenfüllung eine mächtige Schluffschicht, die sich randlich mit den gröberen Einschüttungen des Palten- und Pyhrnbaches verzahnt (Abb. 3). Sie weist auf eine ruhige Sedimentation in einem See im Ennstal hin, in dem wahrscheinlich bei wechselnden Wasserständen die seitlichen Einschüttungen verschieden weit ausgreifen konnten. Auf diese wechselnden Wasserstände weisen auch gering mächtige Torflagen im Sedimentkörper hin.

Diese Sedimentabfolge liegt einer wahrscheinlich im ganzen Ennstal oberhalb des Gesäuses verbreiteten gröberen Kiesschicht auf. Durch die Verzahnung können die Grundwasser aus dem Bereich der Zubringer in die tieferen Grundwasserstockwerke im Ennstal eintreten, wodurch artesisch gespanntes Grundwasser auftritt, das im Raum Selzthal örtlich bis $4 \mathrm{~m}$ über die Oberfläche ansteigt.

Die zur Untersuchung des Grundwasserspeichers im Ennstal von der Ennskraftwerke AG Steyr niedergebrachten Bohrungen ergaben prinzipiell den gleichen Aufbau der hangendsten Sedimentabfolge zwischen Irdning und Gesäuseeingang.

Im Zuge dieser Untersuchungen wurde an 20 Standorten durch Jahre die Spiegelhöhe des gespannten Grundwassers beobachtet. Dabei ergab sich, daß der zuerst knapp unter der Oberfläche liegende Grundwasserspiegel im Bereich Wörschach über das heutige Talniveau ansteigt. Weiter talabwärts nimmt die Höhe ständig bis auf rund $10 \mathrm{~m}$ (bei Admont) über Talniveau zu. Eine genaue Auswertung der Messungen im Vergleich mit den örtlichen meterologischen Bedingungen ergab einen differenzierten Feinablauf der Ganglinien des Grundwassers in Abhängigkeit von örtlichen Niederschlägen und Schneeschmelzen (Platzl 1960) aber im ganzen ein generell geringeres Gefälle der Grundwasseroberfläche als die heutige Enns. 
Eine Deutung der Verhältnisse kann am ehesten dadurch erfolgen, daß die abdichtende oberste Schluffschicht und die mit ihr verzahnten Schwemmkegel in einem See zur Ablagerung kamen, der das Ennstal bis in den Raum Stainach erfüllte. In diesem wurde das hangende, weitgehend das ganze Ennstal hin nach oben abdichtende Schluffpaket mit seinem komplizierten Internaufbau abgelagert. Der Grund dafür, daß unter dieser Schicht kein absolut zusammenhängender Grundwasserkörper in den liegenden gröberen Sedimenten auftritt, liegt wahrscheinlich darin, daß oberhalb der starken seitlichen Einschüttungen kleinere Becken mit Feinsedimenten entstanden, die lokale dichtende Barrieren darstellen.

Der Stau des bis in den Raum Stainach reichenden Sees, dessen Sedimente den Abschluß der Auffüllung des übertieften Bereiches des Ennstales darstellen, muß am Gesäuseeingang erfolgt sein. Im Gegensatz zu Bistritschan (1952) möchte ich den Grund für diesen Rückstau nicht in großen Bergstürzen am Gesäuseeingang, sondern in einer riesigen Massenbewegung suchen.

Bei der Neuaufnahme der Gesäuseberge durch K. BücHNER (1973) stellte sich heraus, daß der Dachsteinkalk der Haindlmauer einen Teil des Reichensteins darstellt, der seit der Gosauzeit nach $\mathrm{N}$ abwanderte und heute auf Werfener Schichten liegt. Da aber der Ennsgletscher zur Würmeiszeit noch ins Gesäuse eindrang, kam es sicher am Gesäuseeingang zu einer nennenswerten Erosion, die nach dem Abschmelzen des Eises zu Bewegungen der Kalkmasse der Haindlmauer geführt haben wird.

Dadurch kam es zu einer ständigen Höherlegung des Gesäuseeinganges und abschließend auch zu einer längerfristigeren Abriegelung als durch grobblockige Bergsturzmassen möglich wäre, während sich die schluffreichen hangendsten Sedimente im Ennstal ausbildeten.

Das Alter des Sees konnte durch palynologische Untersuchungen an Proben aus den Bohrungen für die Pyhrn-Autobahn, die Dr. I. Draxler, Geol. B.-A. durchführte, näher bestimmt werden.

Der Pinus-Wald, der damals an den Hängen des Ennstales stockte, wies eine Waldgrenze in rund $400 \mathrm{~m}$ über dem Tal auf, wie durch den hohen Prozentsatz an Zirbe und das massive Auftreten von Kräutern (Artemisia) angezeigt wird. Ebenso weist das Auftreten von Hippophä̈ auf eine erst kürzlich erfolgte Besiedelung des Rohbodens hin.

Dieses Vegetationsbild spricht für Verhältnisse, wie sie im Vergleich mit dem benachbarten Trauntal (Draxler 1977) das letzte Mal im Alleröd im Raum des Ennstales geherrscht haben können.

\section{Zusammenfassung der Ergebnisse}

Abgesehen von den Gesteins- und topographisch bedingten Stellen am Südende des Attersees und des Salzburger Stammbeckens finden wir im hier dargestellten Raum eine Bindung der großen glazial übertieften Wannen an die Erstreckung des Zehrgebietes des Eisstromnetzes. Am deutlichsten ist diese im Trauntal entwickelt, wo die Gletscherzungen der beiden letzten Eiszeiten kaum über die innerhalb des Alpenkörpers liegenden Becken hinaus vordrangen und es daher nicht mehr zur Ausbildung von Zweigbecken kam.

Ein zweiter Bereich mit langgestreckten, glazial übertieften Wannen findet sich im S des Einzugsgebietes der Traun, die ebenso wie die nördlichen keinen Zusammenhang mit dem Aufbau und den Strukturen des Untergrundes aufweisen. Sie liegen aber, wie eine Detailkartierung zeigte, alle im Zungenbereich kräftiger Lokalgletscher, die während der 
Aufbau- sowie der Abschmelzphase des hochglazialen Würmeisstromnetzes über längere Zeit Bestand hatten. Es scheint sich bei dieser Gletschergröße um eine relativ rasch erreichbare Größenordnung zu handeln, die möglicherweise auch während früherer Eiszeiten öfter auftrat.

Belege für größere, glazial übertiefte Bereiche in gleicher Position konnten kürzlich auch in südlichen Nebentälern des Ennstales gefunden werden. Ob solche auch in den Nebentälern anderer Flußsysteme zu finden sind, wird sich erst durch weitere Aufschlüsse klären lassen.

Diese glazial übertieften Becken zeigen alle die gleiche Form langgestreckter Wannen mit steilen Wänden und einer weitgehend ebenen Sohle, die kaum vom Aufbau des Untergrundes beeinflußt wird. Eine Erklärung für die Entstehung dieser offensichtlich an den Zungenbereich gebundenen Becken kann möglicherweise darin gesehen werden, daß der Gletscher im Bereich der Grenze Nähr- zu Zehrgebiet seine größte Horizontalgeschwindigkeit und damit wohl auch größte Erosionskraft entwickelt, die außerdem noch durch die relative Zunahme des Moränenmaterials im Zuge des Abschmelzens unterstützt wird.

Die Wiederauffüllung begann unmittelbar nach dem Eisrückzug und war selbst in großen Becken wie dem Salzburger Stammbecken in so kurzer Zeit abgeschlossen, daß sich noch keine nennenswerte Vegetation in der Umgebung des Sees entwickeln konnte.

Im Ennstal hingegen dauerte die Sedimentation bedingt durch die ständige Höherlegung des Gesäuseeinganges durch eine Großhangbewegung länger an. Sie war aber, wie die palynologische Untersuchung ergab, bereits knapp nach der Ausbreitung der Gehölzvegetation im Alleröd ebenso beendet. Der durch diesen Mechanismus gegenüber dem prae-mindelzeitlichen Talboden stark verflachte Talverlauf führte zu weitflächigen Sumpfund Moorbildungen, wie sie auch aus anderen Tälern mit ähnlichen Bedingungen ( $\mathrm{z}$. B. Pinzgau) bekannt sind.

Ebenso rasch wurden die großen Deltas im Hallstätter und Traun See aufgeschüttet. $\mathrm{Da}$ aber der Traun im Gegensatz zu den aus Kristallingebieten kommenden Flüssen die hohe Belastung mit Schwebstoffen fehlt, kamen nur gröbere Sedimente zur Ablagerung, die entsprechend ihrer geringen Menge nur verhältnismäßig kleine Areale bedecken.

\section{Schriftenverzeichnis}

Bistritschan, K. (1952): Zur Gelologie der Talauffüllungen des Mitterennstales. - Verh. Geol. B.-A.: 232-235; Wien.

- (1956): Die Talalluvionen des Mitterennstales. - Verh. Geol. B.-A., 184-187; Wien.

Brandecker, H. (1974): Hydrogeologie des Salzburger Beckens. - Steir. Beitr. z. Hydrogeologie, 26: 5-39; Graz.

BüCHNER, K.-H. (1973): Ergebnisse einer geologischen Neuaufnahme der nördlichen und südwestlichen Gesäuseberge (Ober-Steiermark, Osterreich). - Mitt. Ges. Geol. Bergbaustud., 22: 71-95; Wien.

Del-Negro, W., Ebers, E. \& Weinberger, L. (1966): Der pleistozäne Salzachvorlandgletscher. - Veröff. Ges. Bayer. Landesk. e.V., 116 S.; München.

Draxler, I. (1977): Pollenanalytische Untersuchungen von Mooren zur spät- und postglazialen Vegetationsgeschichte im Einzugsgebiet der Traun. - Jahrb. Geol. B.-A., 120: 133-163; Wien.

Gerer, G. (1917): Über die Querverschiebung am Traunsee. - Verh. Geol. R.-A.: 67-69; Wien.

Hasenrodt, K. (1965): Riesengletschertöpfe am Nordausgang des Kalkhochalpen-Durchbruchstales der Salzach bei Golling (Salzburg). - Mitt. Geograph. Ges. München, 50: 161-173; München. 
Husen, D. Van (1968): Ein Beitrag zur Talgeschichte des Ennstales im Quartär. - Mitt. Ges. Geol. Bergbaustud., 18: 249-286; Wien.

- (1977): Zur Fazies und Stratigraphie der jungpleistozänen Ablagerung im Trauntal. Jahrb. Geol. B.-A., 120: 1-130; Wien.

KLAus, W. (1975): Das Mondsee-Interglazial, ein neuer Florenfundpunkt der Ostalpen. - Jahrb. O.Ó. Musealv., 120: 315-344; Linz.

Penck, A. \& Brückner, E. (1909): Die Alpen im Eiszeitalter. - 1: 393 S.; Leipzig (Tauschnitz).

Platzl, M. (1960): Die Grundwasserverhältnisse im mittleren Ennstal. - 100 Jahre Ennsregulierung, Festschr.: 22-29; Wien (Natur und Technik).

Prey, S. (1959): Zwei Tiefbohrungen der Stieglbrauerei in Salzburg. - Verh. Geol. B.-A.: 216224; Wien.

Stummer, E. (1947): Der Aufbau des Salzburger Zungenbeckens. - Mitt. Ges. Salzburger Landeskunde, 86/87: 81-82; Salzburg.

WeINBERGER, L. (1955): Exkursion durch das österreichische Salzachgletschergebiet und die Moränengürtel des Irrsee- und Attersee-Zweiges des Traungletschers. - Verh. Geol. B.-A.: 7-34; Wien. 